Carla Meyer (Mittelalterliche Geschichte), Bernd Schneidmüller (Mittelalterliche Geschichte)

\title{
Zwischen Pergament und Papier
}

In der mediaevistischen Handbuchliteratur wird das lateinische Mittelalter gern als „Pergamentzeitalter“ bezeichnet. ${ }^{1}$ Die Tierhaut war seit dem 8. Jahrhundert nicht nur der dominierende Beschreibstoff ( $\rightarrow$ Pergament), sondern im Blick der Zeitgenossen wie der geschichtswissenschaftlichen Forschung eng mit der häufigsten und weit über Recht und Verfassung hinaus aufschlussreichsten Quellengattung des Mittelalters verknüpft: der Urkunde. Sie dient daher sogar noch häufiger als das Pergament zur Epochenbezeichnung des Urkundenzeitalters.

In den Bezeichnungen als „Pergament-“ oder „Urkundenzeitalter“ schwingt zugleich ein Urteil über den Status von Schrift und Schriftlichkeit dieser Ära mit. Diese wurden - so definiert es Ahasver von Brandt in seinem maßgeblichen Lehrbuch zu den historischen Grundwissenschaften - nämlich über lange Zeit nur eingesetzt, wenn es galt, „das rechtsverbindliche Endprodukt einer Handlung festzulegen“.2 Dies änderte sich erst seit dem Spätmittelalter mit dem Anbruch des „Aktenzeitalters“, in dem eine zunehmend schriftgesteuerte Verwaltung auch Zwischenstadien des administrativen oder geschäftlichen Handelns zu dokumentieren begann. In der Forschung wird dieser Wandel meist pauschal mit der Einführung und Durchsetzung des Papiers in Zusammenhang gebracht. ${ }^{3}$ Die neue Ära der „Akten“ läutete in dieser Vorstellung auch das Ende der Pergamentzeit ein.

Der Beginn der Moderne wird freilich gemeinhin nicht als „Papierzeit“ deklariert. Ihren Auftakt bietet in den Forschungsnarrationen vielmehr das „Druckzeitalter“ ( $\rightarrow$ Typographisch/non-typographisch). So gilt zwar auch das Papier als epochemachend. Der Anbruch der „Papierzeit“ wird in der Mehrzahl entsprechender Publikationen freilich erst ab etwa 1800 datiert. ${ }^{4}$ Anlass dafür ist der explosionsartige, durch neue industrielle Herstellungsverfahren ermöglichte Anstieg des Papierverbrauchs, der nun gesellschaftlich auch breit wahrgenommen und dessen vielgestaltiger Einsatz nicht selten kritisiert wurde.

Doch zwischen „Pergament-“ und „Papierzeit“ klafft in den genannten Definitionen nicht nur eine chronologische Lücke von mindestens 300 Jahren. Die Begriffe

Dieser Beitrag ist im SFB 933 „Materiale Textkulturen“ entstanden, der durch die DFG finanziert wird.

1 Boockmann u. Dormeier 2005 ${ }^{10}$, 185; kritisch Keller 1990, 171f. Zu Pergament und Herrschaft Schneidmüller 2012, 25-45.

2 Von Brandt $2003^{16}, 104$.

3 Vgl. etwa Boockmann u. Dormeier 2005 ${ }^{10}, 185$.

4 Oligmüller 1997, zum Folgenden 33-38; siehe u. a. auch das Kapitel „The Paper Age“ in Müller 2013. 
sind auch insofern irreführend, als beide Beschreibstoffe zeitlich sehr viel früher bzw. länger gebräuchlich waren. Zugleich besaßen sie auch sehr viel früher bzw. länger Bedeutung in den ökonomischen Systemen und der gesellschaftlichen Wertschätzung, als es die genannten Epochenbezeichnungen vermuten lassen. Daraus folgt, dass der postulierte Ablöseprozess viel diffuser erscheinen muss. Dies gilt erstens für das Papier, das in Europa schon seit der Jahrtausendwende bekannt war und sich von Spanien und Italien ausgehend im späteren Mittelalter über alle Teile des Kontinents verbreitete ( $\rightarrow$ Papier). Auch wenn die Mehrheit der Forschung die Rolle des Papiers durch die bereits von den Zeitgenossen als Markstein wahrgenommenen Auswirkungen der „Gutenberg-Revolution“ überstrahlt sieht ( $\rightarrow$ Typographisch/non-typographisch), so taucht in mediaevistischen Studien jedoch zumindest gelegentlich das Epitheton der „Paper Revolution“5 auf. Es verleiht dem nicht nur zeitlich früher einsetzenden, sondern auch in den gängigen Medientheorien bislang meist nur im „Wahrnehmungsschatten“ des Buchdrucks verhandelten Beschreibstoff sein eigenes kulturhistorisches Gewicht. ${ }^{6}$

In der Tat greift es zu kurz, die Durchsetzung des Papiers nur als Begleiterscheinung des Buchdrucks zu deuten. Das stellte der Islamwissenschaftler Jonathan Bloom in seiner Monographie „Paper before Print“ über die Geschichte und Geltung des Papiers in der arabischen Kultur seit dem Frühmittelalter pointiert heraus. ${ }^{7}$ Während der Buchdruck im islamischen Einflussgebiet erst seit dem 18. Jahrhundert breiter etabliert wurde, erschließt Bloom die Bedeutung des schon seit dem 9. Jahrhundert weit verbreiteten Papiers als Beschreibstoff mit transformatorischen Wirkungen in der islamischen Zivilisation. Hier beförderte Papier den Aufbruch literarischer Kreativität auf unterschiedlichen Feldern, von der Theologie über die Naturwissenschaften bis zur Dichtung. ${ }^{8}$ Neben den Wissenschaften berücksichtigte Bloom auch den weitreichenden Einfluss des Papiergebrauchs auf die Administration und damit die Organisation von Herrschaft. Hinzu kam die Einführung papierner Skizzen statt rein mündlicher Übermittlung für einen nachhaltigen Entwicklungsschub im Handwerk, etwa in der Keramikproduktion, der Teppichmanufaktur oder der Architektur.

Eine vergleichbare kulturhistorische Studie zum Papiergebrauch im europäischen Bereich bleibt nach wie vor ein Desiderat. ${ }^{9}$ Schon jetzt ist über die traditionell auf Technik- und Wirtschaftsgeschichte und damit den Herstellungsprozess und den Vertrieb fokussierte Papierforschung abzusehen, dass sich das Papier schon weit vor

5 Siehe u. a. Burns 1981.

6 Zur These, dass die Rolle des Papiers im Vergleich zum Buchdruck in den gängigen Medientheorien „untertheoretisiert“ sei, formulierte Lothar Müller in seinem Abendvortrag „Das Blatt und die Welt. Über die Epoche des Papiers“ (14. November 2013, Universitätsbibliothek Heidelberg) neue Anstöße.

7 Bloom 2001.

8 Bloom 2001, 12.

9 Einen Baustein dazu soll die Heidelberger Habilitationsschrift von Carla Meyer (in Vorbereitung) liefern. 
der „Gutenberg-Revolution“ in einer Vielzahl kultureller Praktiken, Infrastrukturen und Routinen ,einnistete“. ${ }^{10}$ Eindrucksvoll stellt dies Cornel Zwierlein für die Genese des „Nachrichtenbriefs“ dar, dessen Entstehung er in der letzten Dekade des 13. Jahrhunderts verortet: Seine explosionsartige Durchsetzung fällt mit der Etablierung des Papiers zusammen. Alle von Zwierlein - vor allem am Beispiel der europaweiten Korrespondenz der aragonesischen Krone - konsultierten Originalbriefe und Berichte seit den 1290er Jahren wurden auf Papier abgefasst. ${ }^{11}$

Zudem wurde das Pergament an der Schwelle zur Neuzeit nicht einfach vom Papier abgelöst. Mit großer Selbstverständlichkeit blieb die Tierhaut in Europa vielmehr in vielen Bereichen weit über das Mittelalter hinaus in Gebrauch. Grund dafür war das Misstrauen, dass dem Papier europaweit besonders von Herrschaftsträgern bzw. im Bereich von Recht und Ökonomie entgegenschlug. In der Papierforschung sind die frühen Erlasse des 13. Jahrhunderts bekannt, als Kaiser Friedrich II. - nach Robert I. Burns das „,ansonsten doch so innovative ,Staunen der Welt ““12 - Papier als Beschreibstoff für Notariatsinstrumente verbot. ${ }^{13}$

Doch auch in späteren Jahrhunderten wurde diese Reserve vielfach bestätigt. Noch 1784 legte Christian Daniel Anderson in seiner Abhandlung zum Hamburgischen Privatrecht fest, dass die rechtsverbindlichen Originale der „Erb- und Rentbücher“ auf Pergament zu führen seien. ${ }^{14}$ Pergament blieb, wie Hendrik van Huis in einer Studie zur Verwendung von Pergament und Papier in den Stadtverwaltungen Hamburgs und Greifswalds nachweist, gerade für traditionsreiche Amtsbuchserien bis ins frühe 19. Jahrhundert gängiger Beschreibstoff. ${ }^{15}$ Selbst im 20. Jahrhundert wurden politisch wichtige Dokumente wie der Friedensvertrag von Versailles 1919 noch auf Pergament ratifiziert. ${ }^{16}$

Die anhaltende Pergament-Nutzung in der Neuzeit macht ein unterhaltsames Streitgespräch verständlich, in dem der Gelehrte Abraham a Sancta Clara um 1700 die beiden personifizierten Kontrahenten Pergament und Papier um ihre Wertschätzung und ihren Gebrauchswert wetteifern ließ. ${ }^{17}$ Konkrete Zahlen zum Verhältnis der beiden Beschreibstoffe in der Überlieferung wurden bislang noch nicht systematisch erhoben. Einzelne Fallstudien zum ausgehenden 15. Jahrhundert legen jedoch für die anbrechende Ära des Buchdrucks nahe, dass auch die Schriftlichkeit auf Pergament gerade im Bereich der Verwaltung keineswegs abbrach. ${ }^{18}$ Die große Nachfrage nach

10 Müller 2013, 13.

11 Zwierlein 2010, 33.

12 Burns 1981, 1.

13 Huillard-Bréholles 1854, 51 (vor 1226) Nr. 1, sowie 56f. (1231).

14 Anderson 1784, 403.

15 Van Huis 2015.

16 Vgl. Müller 2013, 310.

17 Abraham a Sancta Clara 1695, 49f.

18 Vgl. etwa für das Herzogtum Mailand Ganda 2007, besonders 145f. und 150, mit Verweis auf die 
der Tierhaut führte zugleich dazu, dass in dieser Zeit nicht nur die Papier-, sondern auch die Pergamentproduktion zur Massenfertigung weiterentwickelt worden war. Das zeigen Arnoldo Ganda für die Manufakturen am Gardasee, die u. a. die herzogliche Kanzlei in Mailand belieferten, ${ }^{19}$ oder Emanuela Di Stefano an den hohen Exportzahlen von Pergament aus den Marken. ${ }^{20}$

Umgekehrt ist aber auch der Bedeutungszuwachs des Papiers im späten Mittelalter nicht zu unterschätzen. Der Beschreibstoff muss in Kanzleien und Kontoren weitaus alltäglicher gewesen sein, als es die heutige Archivüberlieferung vermuten lässt. Anders als die zur Rechtssicherung angefertigten Pergamente war die offenbar in hohem Maß ephemere papierne Alltagsschriftlichkeit bis auf wenige Ausnahmen nicht archivwürdig oder wurde in späteren Jahrhunderten kassiert ( $\rightarrow$ Sammeln, Ordnen und Archivieren sowie $\rightarrow$ Beschädigen und Zerstören). Aus diesen erheblich schlechteren Überlieferungschancen ${ }^{21}$ für Papier ergibt sich insbesondere bei der Archivüberlieferung eine massive Verzerrung. Das lässt sich an vereinzelten, meist zufällig erhaltenen Beispielen von ursprünglich sicher massenhaft geführten Gattungen wie etwa Quittungen, Rechnungskladden oder Briefen erkennen. Papier war offenbar schon früh ein „Wegwerfprodukt“, wie Birgit Kata in Studien zu einem der äußerst raren archäologischen Fundkomplexe mit Papier, dem sogenannten Kemptener Mühlberg-Ensemble, vor Augen führt. ${ }^{22}$

Wesentlich positiver sieht die Überlieferungsbilanz von Papier in der Buchkultur aus. Nach einer Studie von Uwe Neddermeyer ${ }^{23}$ verdrängte das Papier schon seit der zweiten Hälfte des 14. Jahrhunderts in weiten Teilen Europas das Pergament bei der Buchherstellung. In der zweiten Hälfte des 15. Jahrhunderts wurden in Süddeutschland und im Alpenraum bereits 85 Prozent aller erhaltenen Codices auf Papier geschrieben. Neddermeyer bringt das Papier auch mit der rasanten Steigerung der Buchproduktion in Verbindung. Nach seinen Ergebnissen fanden solche Zuwächse seit dem frühen 15. Jahrhundert im deutschen Raum nur auf Papier statt. ${ }^{24}$

Diese Hochphase in der Buchproduktion auf Papier korrespondiert mit der gestiegenen Qualität der dafür genutzten Blätter. Insbesondere Bibliotheks- und Restaurierungswissenschaften stellen die in früheren wie späteren Jahrhunderten unerreichte

Massenproduktion der Notare auf Pergament, deren Überlieferung aus dem 15. Jahrhundert im „Fondo Diplomatico“ des Mailänder Staatsarchiv noch heute rund 130.000 Stück umfasst.

19 Ganda 2007, besonders 151-166 mit der Edition entsprechender Belege im Anhang.

20 Di Stefano 2011, 43-72.

21 Begriffsprägung von Esch 1985.

22 Kata 2015.

23 Neddermeyer 1998, besonders 256-263.

24 Älteren Pergamenten wurde in dieser aufblühenden Buchkultur nicht selten zum Verhängnis, dass ihr Materialwert höher als ihr Überlieferungswert galt: So wurden unbeschriebene wie beschriebene, jedoch für nicht länger nützlich erachtete Bögen zweckentfremdet als Makulatur zum Beispiel für die Einbandverstärkung weitergenutzt. 
Güte der Papiere aus dem 15. Jahrhundert heraus. ${ }^{25}$ Auf der Suche nach Gründen für diese ungewöhnliche Beobachtung schlägt der Papierspezialist und „-praktiker“ Timothy Barrett eine zwar noch nicht durch schriftliche Quellen belegte, jedoch auf der Basis seiner Materialanalysen durchaus plausible Hypothese vor: Demnach liege der Erfolg der Papiere aus dem 15. Jahrhundert in der Findigkeit ihrer Hersteller. Sie boten das neue „Substrat“ für Pergament nicht nur in höherer Stückzahl und zu günstigeren Preisen an. Durch technische Innovationen bei der Leimung sei es ihnen vor allem gelungen, die Physiognomie der Papierblätter so weit als möglich den Eigenschaften des Pergaments anzunähern. Nach Barrett war es kein Zufall, dass man dafür auf Pergamentabfälle zurückgriff. Durch die aus ihnen gewonnene Gelatineleimung sei das Papier nicht nur tintenfest geworden. Mit seiner zuvor ungekannten Stabilität und durch seine mit dem Glättstein polierte glänzende Oberfläche musste ein solches Papier als die gelungene Verwirklichung einer Pergamentimitation erscheinen. ${ }^{26}$

\section{Literaturverzeichnis}

Abraham a Sancta Clara (1695): Abraham a Sancta Clara, Judas Der Ertz-Schelm / Für ehrliche Leuth / Oder: Eigentlicher Entwurff / vnd Lebens-Beschreibung deß Iscariotischen Bößwicht, Teil 4, Salzburg.

Anderson (1784): Christian Daniel Anderson, Hamburgisches Privatrecht, Bd. 2, Hamburg. Barrett (2013): Timothy Barrett, „Parchment, Paper and Artisanal Research Techniques“, in: Jonathan Wilcox (Hg.), Scraped, Stroked, and Bound. Materially engaged Readings of Medieval Manuscripts (Utrecht Studies in Medieval Literacy 23), Turnhout, 115-127.

Bloom (2001): Jonathan M. Bloom, Paper Before Print. The History and Impact of Paper in the Islamic World, New Haven.

Boockmann u. Dormeier $\left(2005^{10}\right)$ : Hartmut Boockmann u. Heinrich Dormeier, Konzilien, Kirchen- und Reichsreform 1410-1495 (Gebhardt Handbuch der deutschen Geschichte 8), Stuttgart.

Von Brandt $\left(2003^{16}\right)$ : Ahasver von Brandt, Werkzeug des Historikers. Eine Einführung in die Historischen Hilfswissenschaften, Stuttgart, 175-196.

Burns (1981): Robert I. Burns, „The Paper Revolution in Europe: Crusader Valencia's Paper Industry A Technological and Behavioral Breakthrough“, Pacific Historical Review 50, 1-30.

Di Stefano (2011): Emanuela Di Stefano, Le Marche e Roma nel Quattrocento. Produzioni, mercanti, reti commerciali (Per la storia dell'Università degli Studi di Camerino, Studi e testi 9), Camerino, 43-72.

Esch (1985): Arnold Esch, „Überlieferungschance und Überlieferungszufall als methodisches Problem des Historikers“, Historische Zeitschrift 240, 529-570.

Ganda (2007): Arnaldo Ganda, „La Pergamena a Milano nella Seconda Metà del Quattrocento. Uso, Prezzo, Punti di Vendita e di Fabbricazione“, in: Roberto Guarasci, Anna Rovella u. Raffaella Zaccaria (Hgg.), Scritti in memoria die Raoul Gueze (1926-2005), 145-166.

25 Vgl. u. a. Barrett 2013, 115-127.

26 Barrett 2013, 120. 
Huillard-Bréholles (1854): Jean Louis Alphonse Huillard-Bréholles (Hg.), Historia diplomatica Friderici secundi, Bd. 4.1, Paris.

Van Huis (2015): Hendrik van Huis, „Papier- und Pergamentgebrauch in den Stadtbüchern von Greifswald“, in: Carla Meyer, Sandra Schultz u. Bernd Schneidmüller (Hgg.), Papier im mittelalterlichen Europa. Herstellung und Gebrauch (Materiale Textkulturen 7), Berlin/München/ Boston, 193-214.

Kata (2015): Birgit Kata, „Papier und Pappe im archäologischen Fundspektrum - Bemerkungen zu einer unterschätzten Quellengattung für die Alltagsgeschichte des Mittelalters und der Frühen Neuzeit“, in: Carla Meyer, Sandra Schultz u. Bernd Schneidmüller (Hgg.), Papier im mittelalterlichen Europa. Herstellung und Gebrauch (Materiale Textkulturen 7), Berlin/München/ Boston, 277-307.

Keller (1990): Hagen Keller, „Die Entwicklung der europäischen Schriftkultur im Spiegel der mittelalterlichen Überlieferung. Beobachtungen und Überlegungen“, in: Paul Leidinger und Dieter Metzler (Hgg.), Geschichte und Geschichtsbewußtsein. Festschrift Karl-Ernst Jeismann zum 65. Geburtstag, Münster, 171-204.

Müller (2013): Lothar Müller, Weiße Magie. Die Epoche des Papiers, München.

Neddermeyer (1998): Uwe Neddermeyer, Von der Handschrift zum gedruckten Buch. Schriftlichkeit und Leseinteresse im Mittelalter und in der frühen Neuzeit. Quantitative und qualitative Aspekte, Bd. 1 (Buchwissenschaftliche Beiträge aus dem Deutschen Bucharchiv München 61), Wiesbaden.

Oligmüller (1997): Johannes Georg Oligmüller (Hg.), Papierzeit (Schriften des Rheinischen Industriemuseums 14), Essen.

Schneidmüller (2012): Bernd Schneidmüller, „Imperium und Pergament - Wege zum Heiligen Römischen Reich“, in: Claudia Fabian u. Christiane Lange (Hg.), Pracht auf Pergament. Schätze der Buchmalerei von 780 bis 1180 (Bayerische Staatsbibliothek. Ausstellungskataloge 86), München, 25-45.

Zwierlein (2010): Cornel Zwierlein, „Gegenwartshorizonte im Mittelalter: Der Nachrichtenbrief vom Pergament- zum Papierzeitalter“, Jahrbuch für Kommunikationsgeschichte 12, 3-60. 